\title{
Unnecessary PCls and other stories ...
}

Minerva got her sums wrong in the first item of her column (BMJ 2015;350:h2632, doi:10.1136/bmj.h2632), and the risk of an ischaemic or haemorrhagic stroke at the time of percutaneous coronary intervention is 1.3 per thousand procedures rather than the 13 per thousand stated. (However, the main point still stands despite the lower risk figure.)

Cite this as: BMJ 2015;350:h2940

๑ BMJ Publishing Group Ltd 2015 